\title{
Potential Role of Addiction Pharmacotherapy in Problematic Eating Behavior
}

\author{
Paula J. Edge • Mark S. Gold
}

Published online: 2 March 2014

(C) Springer International Publishing AG 2014

\begin{abstract}
Obesity is pandemic worldwide, and hyperphagia remains extremely difficult for physicians to treat. Currently, appetite suppression continues to be the focus of antiobesity drugs, and these drugs are clearly unsuccessful in the long term. Although the food addiction concept remains controversial, this hypothesis provides a matrix in which to examine disordered eating behaviors and their similarities to addiction. This article looks at food addiction as the high end of an eating disorder continuum, with anorexia nervosa as the low end. Similarities with drug addiction provide an avenue leading to new and potentially more successful treatments.
\end{abstract}

Keywords Anorexia nervosa $\cdot$ Bulimia nervosa .

Binge-eating disorder $\cdot$ Food addiction $\cdot$ Brain reward system . Withdrawal $\cdot$ Comorbidity $\cdot$ Behavioral addictions $\cdot$ Eating disorder treatment

\section{Introduction}

Obesity remains pandemic, and the public continues to spend billions of dollars each year on unsuccessful weight loss schemes. Physicians continue to find successful treatment of hyperphagia to be almost the "impossible dream." Then again, food addiction continues to be a controversial subject in spite of empirical evidence linking the brain and behavioral changes in food addiction to the same changes in drug addiction [1]. Although there are several successful pharmacological

\section{P. J. Edge $(\bowtie) \cdot$ M. S. Gold}

Department of Psychiatry and McKnight Brain Institute, University

of Florida College of Medicine, Suite L4-100 1149 South Newell

Drive, Gainesville, FL 32611, USA

e-mail: pedge@ufl.edu

M. S. Gold

e-mail: msgold@ufl.edu treatments for drug addiction, pharmacotherapy for the management of obesity continues to focus on overall appetite suppression.

\section{Problematic Eating Behavior}

Eating behaviors that fall outside the parameters of the societal norms can be termed problematic or disordered. Disordered eating behaviors range on a continuum from eating extremely small amounts of food to severely overeating. Neurobiological changes, evidenced by brain scans, are expressed in neuropsychological dysfunction. Common eating disorders include anorexia nervosa (AN), bulimia nervosa (BN), and binge-eating disorder (BED). A subset of people with obesity may also be classified as having an eating disorder, i.e., food addiction [2, 3].

A myriad of factors (genetic, social, biological, psychological, and behavioral) interact in a complex and ever-changing dance which can cause eating disorders. [4]. Avena et al. [5, 6] have shown a link between binge eating and obesity in animal studies. Human epidemiological studies also offer significant evidence of a linkage between binge eating and obesity [7-9]. Neuroimaging studies document human brain changes that are nearly identical in subjects viewing pictures of palatable food or drugs of abuse [10].

An overconcern and dissatisfaction with body weight and shape is the common characteristic of AN, BN, and BED. Eating disorders often have a long and relapsing course and have a high rate of comorbid psychiatric disorders. They are very difficult to treat, and especially in AN there is an increased risk of mortality [11].

\section{Anorexia Nervosa}

AN is characterized by very low body weight and an intense fear of getting "fat" or gaining weight. AN can be of restrictive 
type (the person avoids food or eats very little) or purging type. Extreme exercising, laxatives, diuretics, and enemas or vomiting are all purging mechanisms. People with AN have a distorted perception of how their body looks and have persistent lack of recognition of the seriousness of their low body weight [12].

\section{Bulimia Nervosa}

Bulimia is characterized by periods of time (binges) when a significantly larger amount of food is consumed within a short period of time $(2 \mathrm{~h})$ than another individual would typically eat. Loss of control over eating and inability to stop eating are the defining characteristics along with compensatory behaviors following the binge to prevent the gaining of weight. These, as in AN, can be excessive exercising, or purging (vomiting, misuse of laxatives, diuretics, and enemas) [12].

\section{Binge-Eating Disorder}

BED is also characterized by the consumption of large amounts of food in a short time and a loss of control over eating. However, there are no compensatory behaviors present. Eating when not hungry, eating until feeling too full, eating alone to hide the quantity being eaten, an feeling ashamed and guilty over lack of control are all symptoms of BED. The binge-eater feels a significant amount of distress [12].

\section{Food Addiction}

Food addiction has been debated for many years, and its role in obesity is not yet fully defined. Not all obese people are food addicts. The Yale Food Addiction Scale (YFAS) was developed by Gearhardt et al. [2] as a screening instrument and is modeled on the same criteria as drug use disorder: tolerance, withdrawal, loss of control, failed attempts to stop, giving up other important activities owing to use, and emotional distress. Food addiction overlaps with BED symptoms [13]; the YFAS showed internal reliability, good convergent validity, and good discriminant validity. In their publication describing how the YFAS was validated, Gearhardt et al. [14] stated that the YFAS outperformed existing measures in predicting binge-eating behavior. Avena et al. [15•] found overlaps between substance abuse and overeating. They constructed a three-way juxtaposition of DSM-IV criteria for substance abuse to behaviors in animal studies and to clinical accounts of human behavior in BED. In one example, tolerance (needing larger and larger amounts to maintain the same effect) is a classic sign of substance addiction [16]. In animal studies, researchers found escalation of daily sugar intake over a 3-week access period [17]. Correspondingly, in humans, increased food consumption in each binge is often seen as the disorder becomes chronic [18]. The same three-way similarity was found for withdrawal symptoms and other criteria $[15 \bullet$.

\section{Brain Reward Circuitry}

The most decisive roles in the brain's reward system are played by the ventral tegmental area, located in the midbrain, the nucleus accumbens (NAc) which is innervated by the ventral tegmental area, and the prefrontal cortex, which plays a big role in the processes of attention and motivation. The operation of the NAc is dependent on two essential neurotransmitters, dopamine, which promotes desire, and serotonin, which controls satiety and inhibition. Dopamine and serotonin have an inverse relationship: when a stimulus increases the amount of dopamine in the NAc, the amount of serotonin is reduced, and the reverse is also true.

We know that drugs, including alcohol, hijack the reward system. After a period of time, continued use precipitates changes in the brain circuitry; it becomes dysregulated. Dopamine depletion in drug addiction has been known for some time [19]. Studies in both humans and other animals have supported the hypothesis that brain reward circuitry may be dysregulated in some cases of obesity, disordered eating, and more recently, food addiction [20-24]. Reward circuitry activation also regulates food and drug craving behavior [25].

The hypothalamus is recognized as the main center of the brain responsible for regulation of signals for food consumption. Hormones such as leptin, ghrelin, and insulin work on both the hypothalamus and the limbic system within areas such as the caudate nucleus, hippocampus, and insula - the same regions that participate in the control of reward, motivation, learning, emotion, and stress responses [26].

There are two rewards that are considered "natural rewards"; that is, food and sex. Both are essential to the continued existence of all life and were hard-wired into our being. However, food in the natural sense refers to "eating to live." Unfortunately, this has evolved in our modern time into "living to eat" for many people. Just as people may turn to alcohol or drugs as a way to deal with their problems, they may also turn to food. Might eating when stressed, eating when sad or unhappy, and eating when frustrated be how "comfort food" got its name?

\section{Withdrawal}

\section{Alcohol}

In a previous article [27], we wrote on hyperphagia resulting from withdrawal of drugs of abuse. The famous Alcoholics Anonymous acronym is HALT; whenever, I feel Hungry, 
Angry, Lonely, or Tired, I need to HALT, identify, and address this need. Unfortunately, crunchy, salty, sweet, and fatty food can be the means of addressing that need. Many people with alcoholism in recovery show significant weight gain and tend to use food to distract themselves from alcohol craving.

If the craving for alcohol is blunted by ingesting highly palatable food, the alcohol craving is not gone, but is under control and the person with alcoholism is considered to be in recovery. However, take away the ability to access highly palatable food and alcohol craving returns, increasing the risk of relapse. Addiction transference is also a possibility, especially in food addiction [28]. Addiction transference has become commoner and commoner as a consequence of the rising number of bariatric surgical procedures performed [28]. Studies show that in the years following the procedure there is a higher risk of alcohol addiction [29-31].

As a result of the surgery, the absorption rate is changed and a serving of alcohol becomes much more potent. In addition, although the ability to eat a large amount of hyperpalatable food at one time is cut drastically by bariatric surgery, if the patient's food, relationship, and self-image issues are not addressed by therapy, then the risk of addiction transference is higher. Block the craving for addictive food by appetite suppression or bariatric surgery and you might as well spin the wheel of misfortune (see Fig. 1) to see which addiction will take the place of food.

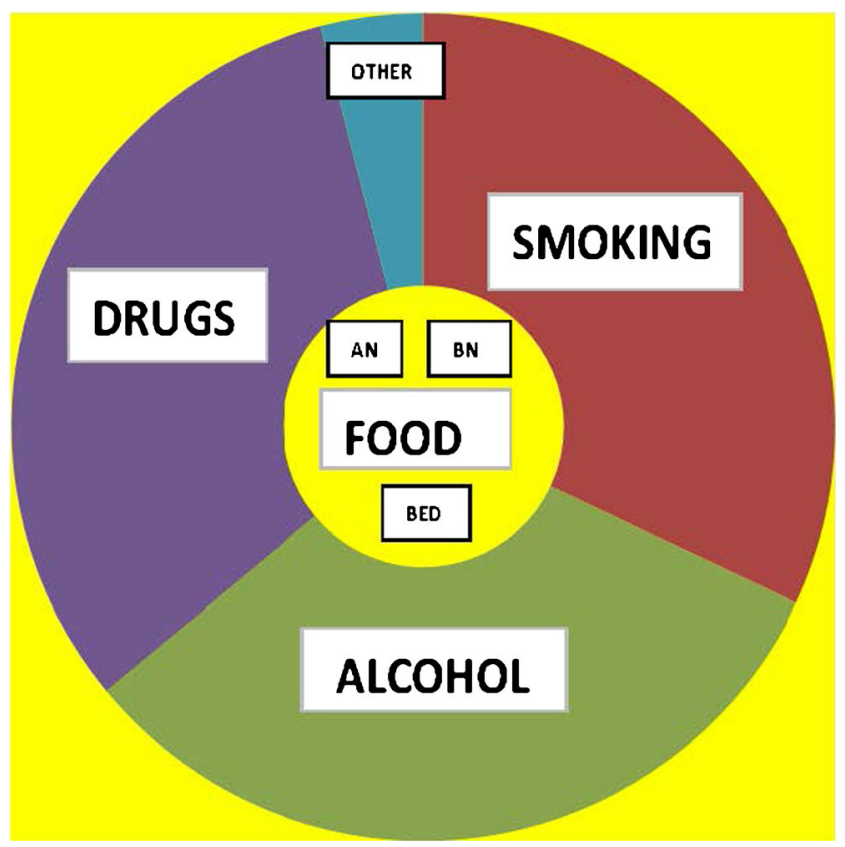

Fig. 1 Food is the solid center surrounded by a moveable wheel of the most popular addictions (drugs, tobacco, alcohol). If any of the addictive substances are blocked, food becomes the addiction. If, however, food is blocked, any of the addictions may occur. Similarly to the game show Wheel of Fortune, you spin the wheel and may not predict which addiction results; ergo "Wheel of Misfortune." $A N$ anorexia nervosa, $B E D$ binge-eating disorder, $B N$ bulimia nervosa
Remembering that the reward and withdrawal systems of the brain are separate entities, use of the addictive substance is mediated by reward rather than withdrawal avoidance. In other words, the substance stimulates its own selfadministration [32], which is more powerful than the desire to avoid withdrawal.

\section{Tobacco}

Many lessons can be learned by looking at tobacco addiction. Tobacco is a good example of a self-perpetuating addiction, even though it is associated with a number of serious medical conditions, including cancers, and has a high rate of psychiatric comorbidities. In 2009, the relationship between BMI and the risk of substance use disorders was examined by Barry and Petry [33]. They found that overweight women were at increased risk of lifetime nicotine dependence, whereas overweight men were at decreased risk. Smoking is associated with decreased appetite and weight loss, and cessation of smoking is associated with increased appetite and weight gain [34].

Smoking has long been used as a weight control method by women with eating disorders. In a study that compared smoking behavior between female community controls and women with eating disorders, higher rates of smoking were found in women with eating disorders, especially those with binge/purge subtypes [35].

\section{Cannabis}

To date there is no effective pharmacological treatment for cannabis use disorder. Marijuana smoking creates strong cravings for and intensifying pleasure in hyperpalatable food, especially sweets and carbs, by stimulating cannabinoid receptors, which increase the drive to eat [36]. It was thought that blocking those receptors would decrease the drive to eat.; the promising drug rimonabant, although successful in animal studies, caused suicidal ideation in humans and was not approved by the FDA [27].

\section{Amphetamines}

These drugs were prescribed for decades for weight loss. Appetite was greatly decreased and energy was greatly increased as were alertness and weight loss. However, amphetamines are highly addictive, and long-term use causes a host of medical issues. Withdrawal from long-term stimulant drug exposure causes dysphoria, anhedonia, irritability, amotivation, and social dysfunction along with rebound hyperphagia and weight gain. Brain changes similar to those seen in trauma-induced brain injury are seen in methamphetamine addicts, and the changes may not ever reverse after withdrawal and abstinence [37]. 


\section{Cocaine}

Cocaine, like amphetamines, inhibits appetite, and withdrawal brings rebound hyperphagia and weight gain. All cocaine is addictive, but crack (a smokeable form of cocaine) is highly addictive compared with sniffed cocaine. As far back at the 1980s we were writing about cocaine abuse and eating disorders [38] and providing evidence that medications successfully used in addictions could also work in some eating disorders $[39,40]$.

\section{Eating Disorders and Withdrawal}

Both $\mathrm{AN}$ and $\mathrm{BN}$ have a high rate of psychiatric comorbidity: lifetime prevalence of substance use disorders is found in $55 \%$ of people with BN and in $23 \%$ of people with $\mathrm{AN}$ $[41,42]$. Studies show that disordered eating also changes the neurocircuitry of the brain [43]. Additionally, the appetiteregulating hormones cortisol and peptide YY (PYY) are associated with psychological and behavioral dysfunction of disordered eating from AN to obesity [44, 45]. Lawson et al. [46] found that higher levels of cortisol and PYY are associated with disordered eating across the weight spectrum in women regardless of BMI. Cortisol and PYY levels are involved in regulating appetite and feeding behavior. In the study of Lawson et al., 65 women participated, divided into four groups (16 with AN, 17 overweight or obese, 12 with normal weight with hypothalamic amenorrhea, and 20 with normal weight in good health). Several hormones were measured, including PYY, leptin, ghrelin, and cortisol. The results showed that no matter which group the women were in, higher levels of cortisol and PYY were associated with disordered eating [46]. These results suggest that specific eating disorders may have abnormalities in appetite regulation. No one knows at this point how this will affect withdrawal of hyperpalatable food in BED or obesity. In a more recent study, Lawson et al. [47] found that increased hypothalamic-pituitary-adrenal drive is associated with decreased appetite and hypoactivation of food motivation neurocircuitry in AN.

Animal studies, reported by Avena et al. [48, 49] among others, have provided strong evidence that excessive sugar consumption operates in a similar manner as consumption of opiates, both in changes to the brain and behavior and in naloxone-precipitated withdrawal symptoms. Some of these symptoms were teeth chattering, tremor, anxiety, aggression, and distress vocalizations [15•]. Interestingly, fat bingeing and sugar bingeing have notable differences in behavior [50]. Sugar bingeing has opiate-like withdrawal symptoms, but fat bingeing does not; sugar bingeing does not affect body weight, but fat bingeing increases body weight. A sugar-fat combination produces addictive-like behaviors (including withdrawal symptoms) and also increases in body weight [51].

Highly palatable food is energy-rich, often consisting of carbs, sugars, fats, or various combinations of these foods. Although animal studies are important, they are not able to incorporate the emotional and cultural components of human binge eating [50]. It is our hypothesis that medications used successfully in treating addiction withdrawal may have a place in easing withdrawal for people with sugar and carb addictions.

\section{A New Approach}

Accepting the food addiction hypothesis as a matrix, our research group has been looking at new approaches to the problem of disordered eating [52]. Avena et al. [53] compared the effects of food restriction and overeating on brain reward systems, finding strong evidence linking overeating with changes in reward-related brain regions. The animal studies of Gold et al. [54, 55] have increased our understanding of food addiction and have provided evidence that drugs used successfully in addiction can be successful in treating overeating. Blum et al. have studied the regulation of food and drug craving behavior by reward circuitry dopaminergic activation [25], along with "liking" versus "wanting" and their links to reward deficiency syndrome [56].

During the same time period, Shriner and Gold $[57,58]$ have been working on procedures to treat the issues of patients with disordered eating. Many of the therapies successfully used in substance abuse treatment, such as cognitive behavioral therapy and motivational interviewing, may also be successful in treating disordered eating.

\section{Conclusion}

It is clear that appetite suppression as a treatment of disordered eating is not successful or able to be maintained over time. Yet both the drug companies and the public continue to search for the "magic pill" that will cure obesity without requiring any lifestyle changes or effort by the patient. Alternatively, some physicians continue to blame the patient as having a lack of willpower. The research is clear: food addiction and obesity are diseases of the brain; this statement is supported by brain imaging.

We recommend research trials combining use of the YFAS to identify food addicts and distinguish them from those with BED, followed by pharmaceutical treatment with medications used successfully in addiction along with therapy using procedures that have also proven to be successful in treating addiction. This three-pronged approach would offer new hope to the public and also to the medical profession. 


\section{Compliance with Ethics Guidelines}

Conflict of Interest Paula J. Edge and Mark S. Gold declare that they have no conflict of interest.

Human and Animal Right and Informed Consent This article does not contain any studies with human or animal subjects performed by either of the authors.

\section{References}

Papers of particular interest, published recently, have been highlighted as:

- Of importance

1. Rossetti C, Spena G, Halfon O, Boutrel B. Evidence for a compulsive-like behavior in rats exposed to alternate access to highly preferred palatable food. Addict Biol. 2013. doi:10.1111/ adb.12065.

2. Gearhardt AN, Corbin WR, Brownell KD. Food addiction: an examination of the diagnostic criteria for dependence. J Addict Med. 2009;3(1):1-7.

3. Gearhardt AN, Corbin WR. The role of food addiction in clinical research. Curr Pharm Des. 2011;17(12):1140-2.

4. Blum K, Oscar-Berman M, Barh D, Giordano J, Gold M. Dopamine genetics and function in food and substance abuse. $\mathrm{J}$ Genet Syndr Gene Ther. 2013. doi:10.4172/2157-7412.1000121.

5. Avena N, Rada P, Bocarsly ME, Hoebel BG. Binge eating as a form of addiction: evidence from an animal model of sugar addiction. In: Chambers N, editor. Binge eating: psychological factors, symptoms and treatment. New York: Nova; 2009. p. 95-123.

6. Avena NM. Food and addiction: implications and relevance to eating disorders and obesity. Curr Drug Abuse Rev. 2011;4(3): $131-2$.

7. Barry D, Grilo CM, Masheb RM. Comparison of patients with bulimia nervosa, obese patients with binge eating disorder, and nonobese patients with binge eating disorder. J Nerv Ment Dis. 2003;191(9):589-94.

8. Berner LA, Bocarsly ME, Hoebel BG, Avena NM. Pharmacological interventions for binge eating: lessons from animal models, current treatments, and future directions. Curr Pharm Des. 2011;17(12):1180-7.

9. Gearhardt AN, White MA, Masheb RM, Grilo CM. An examination of food addiction in a racially diverse sample of obese patients with binge eating disorder in primary care settings. Compr Psychiatry. 2013;54(5):500-5. doi:10.1016/j.comppsych.2012.12. 009.

10. Volkow ND, Wang GJ, Fowler JS, Tomasi D, Baler R. Food and drug reward: overlapping circuits in human obesity and addiction. Curr Top Behav Neurosci. 2012;11:1-24. doi:10.1007/7854_2011_169.

11. Herzog DB, Eddy KT. Eating disorders: what are the risks? J Am Acad Child Adolesc Psychiatry. 2009;48(8):782-3. doi:10.1097/ CHI.0b013e3181aa03d7.

12. American Psychiatric Association. Diagnostic and statistical manual of mental disorders. 5th ed. Washington: American Psychiatric Association; 2013.

13. Avena NM. The study of food addiction using animal models of binge eating. Appetite. 2010;55(3):734-7.

14. Gearhardt AN, Corbin WR, Brownell KD. Preliminary validation of the Yale Food Addiction Scale. Appetite. 2009;52(2):430-6.
15. Avena NM, Bocarsly ME, Hoebel BG, Gold MS. Overlaps in the nosology of substance abuse and overeating: the translational implications of "food addiction". Curr Drug Abuse Rev. 2011;4(3): 133-9. This article discusses what happens when the brain pathways for motivation and reinforcement become dysregulated and the neurochemical evidence of reward-related brain dysfunctions in $B E D, B N$, and $A N$.

16. American Psychiatric Association. Diagnostic and statistical manual of mental disorders. 4th ed. Washington: American Psychiatric Association; 2000.

17. Colantuoni C, Schwenker J, McCarthy J, Rada P, Ladenheim B, Cadet JL, et al. Excessive sugar intake alters binding to dopamine and mu-opioid receptors in the brain. Neuroreport. 2001;12(16): $3549-52$.

18. Davis C, Carter JC. Compulsive overeating as an addiction disorder. A review of theory and evidence. Appetite. 2009;53(1):1-8.

19. Dackis CA, Gold MS. New concepts in cocaine addiction: the dopamine depletion hypothesis. Neurosci Bio Rev. 1985;9:469-77.

20. Avena NM, Gold JA, Kroll C, Gold MS. Further developments in the neurobiology of food and addiction: update on the state of the science. Nutrition. 2012;28(4):341-3. doi:10.1016/j.nut.2011.11. 002.

21. Avena NM, Bocarsly ME. Dysregulation of brain reward systems in eating disorders: neurochemical information from animal models of binge eating, bulimia nervosa, and anorexia nervosa. Neuropharmacology. 2012;63(1):87-96. doi:10.1016/j.neuropharm. 2011.11.010.

22. Stice E, Yokum S, Zald D, Dagher A. Dopamine-based reward circuitry responsivity, genetics, and overeating. Curr Top Behav Neurosci. 2011;6:81-93. doi:10.1007/7854_2010_89.

23. Johnson PM, Kenny PJ. Dopamine D2 receptors in addiction-like reward dysfunction and compulsive eating in obese rats. Nat Neurosci. 2010;13(5):635-41.

24. Gearhardt AN, Yokum S, Orr PT, Stice E, Corbin WR, Brownell KD. Neural correlates of food addiction. Arch Gen Psychiatry. 2011;68(8):808-16. doi:10.1001/archgenpsychiatry.2011.32.

25. Blum K, Liu Y, Shriner R, Gold MS. Reward circuitry dopaminergic activation regulates food and drug craving behavior. Curr Pharm Des. 2011;17(12):1158-67.

26. Volkow ND, Wang GJ, Fowler JS, Telang F. Overlapping neuronal circuits in addiction and obesity: evidence of systems pathology. Philos Trans R Soc Lond B. 2008;363(1507):3191-200.

27. Edge PJ, Gold MS. Drug withdrawal and hyperphagia: lessons from tobacco and other drugs. Curr Pharm Des. 2011;17(12):1173-9.

28. Blum K, Bailey J, Gonzalez AM, Oscar-Berman M, Liu Y, Giordano J, Braverman E, Gold M Neuro-genetics of reward deficiency syndrome (Rds) as the root cause of "addiction transfer": a new phenomenon common after bariatric surgery. J Genet Syndr Gene Ther. 2011. doi:10.4172/2157-7412.S2-001.

29. Conason A, Teixeira J, Hsu CH, Puma L, Knafo D, Geliebter A. Substance use following bariatric weight loss surgery. JAMA Surg. 2013;148(2):145-50. doi:10.1001/2013.jamasurg.265.

30. Hajnal A, Zharikov A, Polston JE, Fields MR, Tomasko J, Rogers $\mathrm{AM}$, et al. Alcohol reward is increased after Roux-en-Y gastric bypass in dietary obese rats with differential effects following ghrelin antagonism. PLoS One. 2012;7(11):e49121. doi:10.1371/ journal.pone.0049121.

31. King WC, Chen JY, Mitchell JE, Kalarchian MA, Steffen KJ, Engel $\mathrm{SG}$, et al. Prevalence of alcohol use disorders before and after bariatric surgery. JAMA. 2012;307(23):2516-25. doi:10.1001/ jama.2012.6147.

32. Dupont R, Gold MS. Withdrawal and reward: implications for detoxification and relapse prevention. Psychiatr Ann. 1995;25: 663-8.

33. Barry D, Petry NM. Associations between body mass index and substance use disorders differ by gender: results from the National 
Epidemiologic Survey on Alcohol and Related Conditions. Addict Behav. 2009;34(1):51-60. doi:10.1016/j.addbeh.2008.08.008.

34. Shriner RL, Graham NA, Gold MS. Smoking cessation and the risk for type 2 diabetes mellitus. Ann Intern Med. 2010;152(11):755, author reply 755-6.

35. Anzengruber D, Klump KL, Thornton L, Brandt H, Crawford S, Fichter MM, et al. Smoking in eating disorders. Eat Behav. 2006;7(4):291-9.

36. Kirkham TC. Cannabinoids and appetite: food craving and food pleasure. Int Rev Psychiatr. 2009;21(2):163-71. doi:10.1080/ 09540260902782810.

37. Gold MS, Kobeissy FH, Wang KK, Merlo LJ, Bruijnzeel AW, Krasnova IN, et al. Methamphetamine- and trauma-induced brain injuries: comparative cellular and molecular neurobiological substrates. Biol Psychiatry. 2009;66(2):118-27. doi:10.1016/j. biopsych.2009.02.021.

38. Jonas JM, Gold MS. Cocaine abuse and eating disorders. Lancet. 1986;1(8477):390-1.

39. Jonas JM, Gold MS. Naltrexone reverses bulimic symptoms. Lancet. 1986;1(8484):807.

40. Jonas JM, Gold MS. The use of opiate antagonists in treating bulimia: a study of low-dose versus high-dose naltrexone. Psychiatry Res. 1988;24(2):195-9.

41. Franko DL, Dorer DJ, Keel PK, et al. How do eating disorders and alcohol use disorder influence each other? Int J Eat Disord. 2005;38:200-7.

42. Franko DL, Dorer DJ, Keel PK, Jackson S, Manzo MP, Herzog DB. Interactions between eating disorders and drug abuse. J Nerv Ment Dis. 2008;196(7):556-61.

43. Frank GK, Bailer UF, Henry S, Wagner A. Kaye WH Neuroimaging studies in eating disorders. CNS Spectr. 2004;9(7): 539-48.

44. Bailer UF, Kaye WH. A review of neuropeptide and neuroendocrine dysregulation in anorexia and bulimia nervosa. Curr Drug Targets CNS Neurol Disord. 2003;2(1):53-9.

45. Bailer UF, Price JC, Meltzer CC, Mathis CA, Frank GK, Weissfeld $\mathrm{L}$, et al. Altered $5-\mathrm{HT}_{2 \mathrm{~A}}$ receptor binding after recovery from bulimia-type anorexia nervosa: relationships to harm avoidance and drive for thinness. Neuropsychopharmacology. 2004;29(6):1143-55.

46. Lawson EA, Eddy KT, Donoho D, Misra M, Miller KK, Meenaghan E, et al. Appetite-regulating hormones cortisol and peptide YY are associated with disordered eating psychopathology, independent of body mass index. Eur J Endocrinol. 2011;164(2): 253-61. doi:10.1530/EJE-10-0523.

47. Lawson EA, Holsen LM, Desanti R, Santin M, Meenaghan E, Herzog DB, et al. Increased hypothalamic-pituitary-adrenal drive is associated with decreased appetite and hypoactivation of foodmotivation neurocircuitry in anorexia nervosa. Eur J Endocrinol. 2013;169(5):639-47. doi:10.1530/EJE-13-0433.

48. Avena NM, Gold MS. Food and addiction - sugars, fats and hedonic overeating. Addiction. 2011;106(7):1214-5. doi:10.1111/j.13600443.2011.03373.x, discussion 1219-20.

49. Colantuoni C, Rada P, McCarthy J, Patten C, Avena NM, Chadeayne A, et al. Evidence that intermittent, excessive sugar intake causes endogenous opioid dependence. Obes Res. 2002;10(6):478-88.

50. Avena NM, Rada P, Hoebel BG. Sugar and fat bingeing have notable differences in addictive-like behavior. J Nutr. 2009;139(3):623-8. doi:10.3945/jn.108.097584.

51. Berner LA, Avena NM, Hoebel BG. Bingeing, self-restriction, and increased body weight in rats with limited access to a sweet-fat diet. Obesity (Silver Spring). 2008;16(9):1998-2002.

52. Avena NM, Murray S, Gold MS. The next generation of obesity treatments: beyond suppressing appetite. Front Psychol. 2013;4: 721. doi:10.3389/fpsyg.2013.00721.

53. Avena NM, Murray S, Gold MS. Comparing the effects of food restriction and overeating on brain reward systems. Exp Gerontol. 2013;48(10):1062-7. doi:10.1016/j.exger.2013.03.006.

54. Gold MS, Avena NM. Animal models lead the way to further understanding food addiction as well as providing evidence that drugs used successfully in addictions can be successful in treating overeating. Biol Psychiatry. 2013;74(7):e11. doi:10.1016/j. biopsych.2013.04.022.

55. Yarnell S, Oscar-Berman M, Avena N, Blum K, Gold M. Pharmacotherapies for overeating and obesity. J Genet Syndr Gene Ther 2013. doi:10.4172/2157-7412.1000131.

56. Blum K, Gardner E, Oscar-Berman M, Gold M. "Liking" and "wanting" linked to reward deficiency syndrome (RDS): hypothesizing differential responsivity in brain reward circuitry. Curr Pharm Des. 2012;18(1):113-8.

57. Shriner RL, Gold MS. Treatment of overeating and obesity as an addiction. Dir Psychiatry. 2010;30(4):189-203.

58. Shriner RL, Gold MS. Current and future treatments of obesity. Dir Psychiatry. 2012;32(1):53-65. 